\title{
Transition from stress-driven to thermally activated stress relaxation in metallic glasses
}

\author{
J. C. Qiao, ${ }^{1}$ Yun-Jiang Wang,,${ }^{2,3, *}$ L. Z. Zhao, ${ }^{4}$ L. H. Dai, ${ }^{2,3}$ D. Crespo, ${ }^{5}$ J. M. Pelletier, ${ }^{6}$ L. M. Keer, ${ }^{7}$ and Y. Yao ${ }^{1, \dagger}$ \\ ${ }^{1}$ School of Mechanics, Civil Engineering and Architecture, \\ Northwestern Polytechnical University, Xi'an 710072, China \\ ${ }^{2}$ State Key Laboratory of Nonlinear Mechanics, Institute of Mechanics, Chinese Academy of Sciences, Beijing 100190, China \\ ${ }^{3}$ School of Engineering Science, University of Chinese Academy of Sciences, Beijing 101408, China \\ ${ }^{4}$ Institute of Physics, Chinese Academy of Science, Beijing 100190, P. R. China \\ ${ }^{5}$ Departament de Física, EETAC, Universitat Politècnica de Catalunya, 08860-Castelldfels, Barcelona, Spain \\ ${ }^{6}$ Université de Lyon, MATEIS, UMR CNRS5510, Bat. B. Pascal, INSA-Lyon, F-69621 Villeurbanne cedex, France \\ ${ }^{7}$ Department of Mechanical Engineering, Northwestern University, 2145 Sheridan Rd., Evanston, IL60208, USA
}

(Dated: August 15, 2016)

\begin{abstract}
The short-range ordered, but long-range disordered structure of metallic glasses yields strong structural and dynamic heterogeneities. Stress relaxation is a technique to trace the evolution of stress in response to a fixed strain, which reflects the dynamic features phenomenologically described by the Kohlrausch-Williams-Watts (KWW) equation. The KWW equation describes a broad distribution of relaxation times with a small number of empirical parameters, but it does not arise from a particular physically motivated mechanistic picture. Here we report an anomalous two-stage stress relaxation behavior in a $\mathrm{Cu}_{46} \mathrm{Zr}_{46} \mathrm{Al}_{8}$ metallic glass over a wide temperature range and generalize the findings in other compositions. Thermodynamic analysis identifies two categories of processes: a fast stress-driven event with large activation volume and a slow thermally activated event with small activation volume, which synthetically dominates the stress relaxation dynamics. Discrete analyses rationalize the transition mechanism induced by stress and explain the anomalous variation of the KWW characteristic time with temperature. Atomistic simulations reveal that the stress-driven event involves virtually instantaneous short-range atomic rearrangement, while the thermally activated event is the percolation of the fast event accommodated by the long-range atomic diffusion. The insights may clarify the underlying physical mechanisms behind the phenomenological description and shed light on correlating the hierarchical dynamics and structural heterogeneity of amorphous solids.
\end{abstract}

Subject Areas: Materials Science, Nonlinear Dynamics, Computational Physics

\section{INTRODUCTION}

As a relatively new family of amorphous materials, metallic glasses (MGs) exhibit excellent physical and chemical properties. MGs are usually obtained by rapid quenching from their melts, and show distinctive mechanical behaviors beyond the conventional oxide and polymer glasses due to the unique dense packing of disordered atomic structure [1-4]. Structural inhomogeneity [5-10] usually leads to dynamic heterogeneity [11-15] which is closely correlated with the viscoelasticity and plasticity of MGs [16], both facts of engineering importance and scientific curiosity. Unraveling the atomic-scale structure-dynamic relationship is a paramount challenge [1722] as well as a pressing necessity towards eventual applications of MGs [23].

Stress relaxation is a technique to trace the temporal variation of stress in response to a fixed magnitude of strain generated in the structure, thus reflecting the characteristics of viscoelastic and plastic deformation of the material. It is an important clue to link the dynamic responses to the intrinsic atomic-level structure, which in turn determines the thermodynamic status (aged or rejuvenated) [24, 25] and consequently the macroscopic mechanical performance of MGs [26-29]. Considerable research has been done on the stress

\footnotetext{
* yjwang@imech.ac.cn

$\dagger$ yaoy@nwpu.edu.cn
}

relaxation nature of various MGs [30-34], which demonstrated that localized plastic flow could be activated during the viscoelastic and plastic deformation induced by stress relaxation. Stress relaxation induces a simultaneous excitation at all timescales, thus allowing one to understand the fundamental science behind the potential applications as an engineering material [30-34]. However, the understanding of the microscopic mechanisms accommodating the stress relaxation in amorphous alloys is limited. In particular, the physical connection between the macroscopic stress relaxation behavior and the structural relaxation (primary $\alpha$, and secondary $\beta$ ) usually explored by the internal friction experiment remains quite mysterious and elusive [22, 35, 36].

The whole relaxation spectrum of viscoelastic materials is commonly fitted to the empirical stretched exponential Kohlrausch-Williams-Watts (KWW) function [30-34]. However, the stretched exponential is only a phenomenological description of the behavior and does not arise from a particular physically motivated mechanistic picture. It describes a broad distribution of relaxation times, but with a small number of empirical fitting parameters to describe the distribution. Here, experiments uncover an anomalous but universal two-stage stress relaxation in a prototypical $\mathrm{Cu}_{46} \mathrm{Zr}_{46} \mathrm{Al}_{8}$ glassy system. Thermodynamic and discrete analysis on the stress relaxation spectra rationalize and explain the transition from fast stress-driven to slow thermally activated mechanisms with stress decreasing in stress relaxation. Atomic simulations clarify the atomic-scale mechanisms accommodating stress relaxation and demonstrate their competition. The com- 
prehensive insights from combined experiment, theory, and simulations drive one to reexamine the complex correlation between stress, energetic, and structural relaxations of amorphous alloys, and may rationalize the physical mechanisms underlying the empirical KWW function.

\section{EXPERIMENTS AND THEORY}

\section{A. Stress relaxation experiment}

Benefitting from the excellent thermal stability of the $\mathrm{CuZr}$ based MG, in our work, MG ribbons (thickness $~ 30 \mu \mathrm{m}$ and width $\sim 1 \mathrm{~mm}$ ) with atomic composition $\mathrm{Cu}_{46} \mathrm{Zr}_{46} \mathrm{Al}_{8}$ were processed by the melt-spinning technique in an inert argon atmosphere. The thermal properties of the samples were determined by differential scanning calorimetry (DSC), where the glass transition temperature is $697 \mathrm{~K}$ at a heating rate of 20 $\mathrm{K} / \mathrm{min}$.

The tensile stress relaxation experiments were performed using a TA Q800 dynamic mechanical analyzer (DMA). In order to rule out any influences from initial states, the MG ribbons were heated above the glass transition temperature prior to the measurements. The tensile stress relaxation, carried out at a constant strain of $0.4 \%$ was loaded on the model alloy for 30 minutes after an initial 3 min equilibration.

\section{B. Discrete analysis}

In order to determine the presence of discrete relaxation times, the relaxation response was fitted to a distribution of discrete relaxation times given by

$$
\sigma(t) / \sigma_{0}=\sum_{i=0}^{n} a_{i} \exp \left(-\frac{t}{\tau_{i}}\right)=a_{0} \delta(t)+\sum_{i=1}^{n-1} a_{i} \exp \left(-\frac{t}{\tau_{i}}\right)+a_{\infty}
$$

The relaxation times, namely 0 - instantaneous relaxation -, $\tau_{i}$ and $\infty$ - purely elastic response - are fixed. We fit a single functional form depending on a discrete intensity coefficient $a(\tau)$. This is a variational fitting method using non-orthogonal fitting functions, which is only convergent with very small relative tolerances. In order to span it on a proper timescale, the successive relaxation times were chosen in logarithmic ratio, that is $\frac{\tau_{i}}{\tau_{-1 i}}=c t$. Thus the fitting parameters are only the $n+1$ non-negative intensity coefficients, $a_{0} \ldots a_{n}$. The minimization was performed by using the sequential quadratic programming method implemented in Maple [37], with $\varphi=1.1$ and $n=151$, and a relative tolerance lower than $10^{-11}$. The smooth form of the fitting functions and the non-negativity of the $a_{i}$ coefficients allowed us to obtain a low number, physically significant coefficients corresponding to the discrete relaxation times.

\section{Atomistic simulations}

The MD stress relaxation tests were conducted by LAMMPS [38]. A model glass of $\mathrm{Cu}_{46} \mathrm{Zr}_{46} \mathrm{Al}_{8}$ with 13,500 atoms was prepared by a heating-quenching technique with a cooling rate of $10^{10} \mathrm{~K} / \mathrm{S}$. The force field was described by an embedded-atom method (EAM) potential [39]. To simulate the relaxation tests, we first pulled the simulation box to the tensile strain of $5 \%$ (the yield strain is $6.4 \%$ ) with a constant strain rate $10^{8} \mathrm{~s}^{-1}$, and then fixed the uniaxial strain to trace the time evolution of stress tensor for $50 \mathrm{~ns}$. The simulations were carried out with an isothermal-isobaric ensemble. The visualization is performed by OVITO [40].

\section{RESULTS}

\section{A. Anomalous stress relaxation at low temperature}

The isothermal stress relaxation spectra of $\mathrm{Cu}_{46} \mathrm{Zr}_{46} \mathrm{Al}_{8}$ MG, obtained at increasing temperatures from $393 \mathrm{~K}$ to 693 $\mathrm{K}$, are displayed in Fig. 1(a). The relaxation curves were fitted to a phenomenological KWW equation for the entire temperature range. The KWW equation can characterize the evolution of stress as function of time during the stress relaxation test, i.e.

$$
\sigma(t)=\sigma_{\infty}+\sigma_{0} \exp \left[-\left(\frac{t}{\tau_{\mathrm{c}}}\right)^{\beta_{\mathrm{KWw}}}\right]
$$

where $\sigma_{0}$ is the initial stress, $\sigma_{\infty}$ a relaxed reference stress at infinite time. Closely related to the structural heterogeneity of glass [30], $\beta_{\mathrm{KWW}}$ is a stretched exponent parameter, and $\tau_{\mathrm{c}}$ is a characteristic time that usually separates different relaxation mechanisms [34]. By using the empirical KWW model to fit the stress relaxation data we can compare with previous results and show a qualitative trend of the mechanism transition by tracing the variation of the effective relaxation time $\tau_{\mathrm{c}}$ with change in temperature.

The fitted parameters as a function of temperature are shown in Fig. 1(b). The parameter $\beta_{\mathrm{KWw}}$ lies within [0.4, 1] as usually found in the stress relaxation of amorphous alloys $[30,33,34]$. However, the characteristic time $\tau_{\mathrm{c}}$ shows an unexpected behavior, first increasing and then decreasing with increasing temperature, with a critical temperature of $450 \mathrm{~K}$. This is contradictory to the common sensing that relaxation time decreases with increasing temperature for a specific relaxation mechanism. Similar experimental results have been observed in other typical metallic glasses (see Fig. S1 [41]). To examine the anomalous relaxation behaviors, we provide the original stress relaxation spectra of three low temperature cases in Fig. 1(c) where it is obvious that the stress relaxation at low temperature can be divided into two stages: a fast relaxation and slow relaxation process, with a boundary at $\sim 200$ seconds. The fast and slow relaxation may indicate different relaxation mechanisms during stress relaxation. 

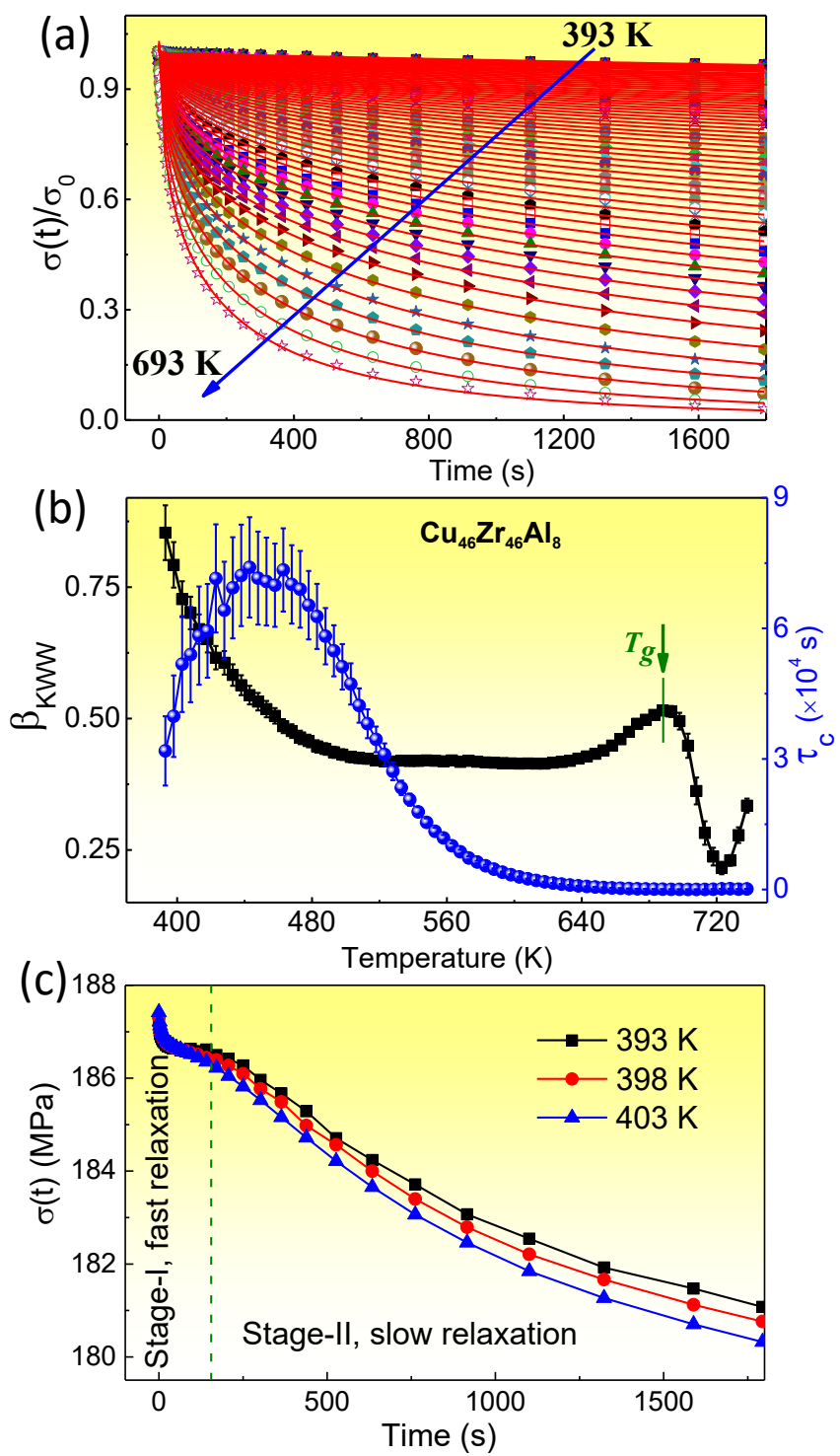

FIG. 1. (Color online) Stress relaxation of a $\mathrm{Cu}_{46} \mathrm{Zr}_{46} \mathrm{Al}_{8}$ MG. (a) Stress-relaxation spectra from 393 to $693 \mathrm{~K}\left(T_{\mathrm{g}}=697 \mathrm{~K}\right)$. The stress levels have been normalized by the initial stresses. The red lines denote the phenomenological KWW fittings. (b) The fitted KWW parameters $\beta_{\mathrm{KWw}}$ and $\tau_{\mathrm{c}}$ as a function of temperature. Variation of $\tau_{\mathrm{c}}$ with $T$ indicates an anomalous stress-relaxation regime at low temperatures. (c) Original stress-relaxation curves demonstrate clearly two-stage relaxation, i.e., fast and slow relaxation, at low temperatures.

\section{B. Thermodynamic analyses}

We use a strategy of thermodynamic analysis to characterize the fast and slow events, which are commonly believed to be thermally activated, and stress-driven rate-controlling processes during stress relaxation (detailed theory in Fig. S2 [41]) $[42,43]$. The results are summarized in Fig. 2. In Fig. 2(a), the logarithmic minus stress rate is plotted against stress at different temperatures. We plot it in this way since the slope of $\ln (-\dot{\sigma})$ with respect to $\sigma$ is related to the activation volume of an event as

$$
\Omega=\sqrt{3} k_{\mathrm{B}} T \frac{\partial \ln (-\dot{\sigma})}{\partial \sigma} .
$$

Here $\Omega$ is the activation volume which is proportional to the size of the event, $T$ temperature, and $k_{\mathrm{B}}$ Boltzmann constant. As shown in Fig. 2(a), the slope changes sharply from high to low stress with a critical magnitude at around $186.5 \mathrm{MPa}$ (the critical stress and time of mechanism transition are shown in Fig. S3 [41]). The transition is particularly pronounced at low temperature, as it is exemplified in the case of $403 \mathrm{~K}$ shown in the inset of Fig. 2(a). The fast event takes place at the early stage of the stress relaxation process with higher stress level, while the slow event occurs at a lower stress. The calculated activation volumes of the two events are shown in Fig. 2(b) as a function of temperature. A noted reasonable trend is that the activation volumes decrease with increasing temperature, which means that the relaxation event becomes less collective at higher temperature. An interesting fact is that the fast event has a very large activation volume, of the order of $\sim 100 \mathrm{~nm}^{3}$ at low temperature and decreases rapidly with increasing temperature. But the slow event has a much smaller activation volume of the order of $1 \mathrm{~nm}^{3}$, and decreases slightly with increasing temperature compared with that of the fast event. The inset of Fig. 2(b) shows the ratio of activation volumes of the fast and slow event. We find that the ratio decreases with increasing temperature, and becomes nearly constant above a critical temperature of approximately $450 \mathrm{~K}$, which corresponds to the critical temperature of the KWW parameter $\tau_{\mathrm{c}}$ as shown in Fig. 1(b) and may indicate that the fast event dominates stress relaxation at the initial stage, while the slow event governs the later one. Finally, there seems to be left a puzzle that a negative activation volume might appear in the transition region of the two categories of events, in particular at low temperature, as shown by the inset of Fig. 2(a). It is not intuitive since a thermally-activated, and stress-driven rate-controlling physical process should have a positive activation volume $[44,45]$. Actually, this is an artifact since it is not reasonable anymore to deduce the activation volume using Eq. (3) if two or more processes are strongly coupled and hard to decompose.

Furthermore, the activation enthalpies $\Delta H(\sigma)=\Delta Q-$ $(\sigma / \sqrt{3}) \Omega$ of the two events at specific stresses are determined by the Arrhenius plot of $\ln (-\dot{\sigma})$ against inverse temperature, as shown in Fig. 2(c-d), respectively. The unbiased activation energy is $\Delta Q$ in the case of $\sigma=0$. For the fast event, the activation enthalpy is $0.74 \mathrm{eV}$ at $187 \mathrm{MPa}$, while it is $0.88 \mathrm{eV}$ at $155 \mathrm{MPa}$ for the slow event. Considering that the activation volume at $550 \mathrm{~K}$ is about $3 \mathrm{~nm} 3$, the unbiased activation energy of the big event is of the order of $\Delta Q=\Delta H(\sigma)+(\sigma / \sqrt{3}) \Omega=2.76 \mathrm{eV}$, which is about $46 k_{\mathrm{B}} T_{\mathrm{g}}$ $\left(T_{\mathrm{g}}=697 \mathrm{~K}\right)$ for $\mathrm{Cu}_{46} \mathrm{Zr}_{46} \mathrm{Al}_{8}$. Thus the activation free energy is significantly reduced by the work done by the stress $(\sigma / \sqrt{3}) \Omega$. The fast event is actually a stress-driven process occurring at the initial stage of the stress relaxation (see Fig. S4 for the sketch of stress-driven, and thermally activated processes). However, the slow event has an activation energy of $1.2 \mathrm{eV}$, considering the activation volume of $0.7 \mathrm{~nm}^{3}$ at 570 

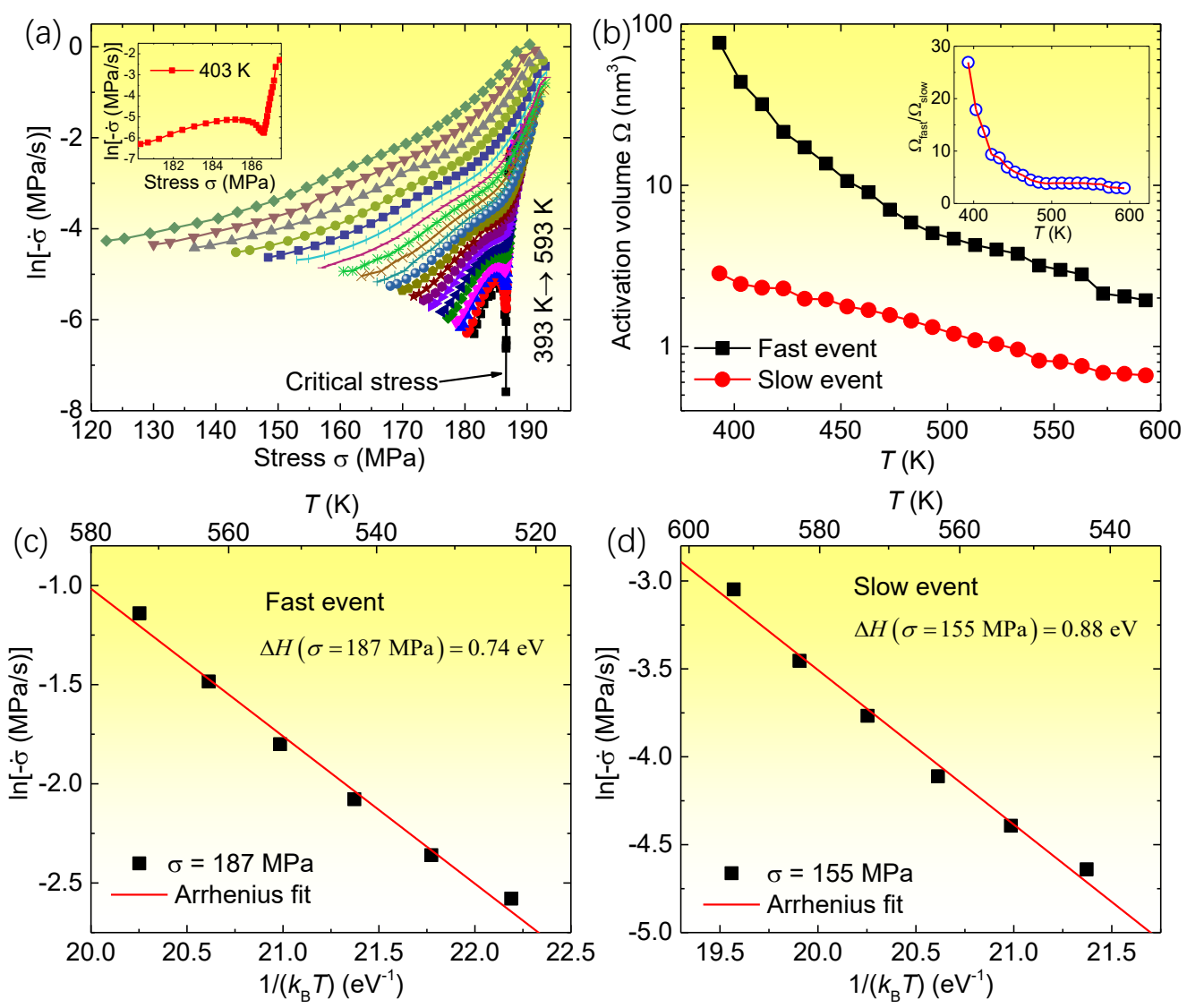

FIG. 2. (Color online) Transition from stress-driven to thermally activated stress relaxation. (a) Dependence of $\ln (-\dot{\sigma})$ on stress. The inset shows an example at $403 \mathrm{~K}$, which indicates a clear transition of relaxation mechanisms with variation in stress. (b) The derived activation volume of the fast and slow events versus temperature. The inset shows the ratio between them. (c and d) Arrhenius plots indicates the fast event presents an activation enthalpy of $0.74 \mathrm{eV}$ at $187 \mathrm{MPa}$; while it is $0.88 \mathrm{eV}$ at $155 \mathrm{MPa}$ for the slow event.

$\mathrm{K}$. The activation energy is about $22 k_{\mathrm{B}} T_{\mathrm{g}}$, which is of the order of the activation energy for the usual $\beta$ relaxation (Fig. S5 [41]) in MGs [35, 46]. Considering the relatively small activation volume, the free energy barrier is overcome mainly by the thermal energy instead of stress. The slow event is mainly a thermally activated process which takes place on a longer time-scale. There exists a transition from the stress-driven to thermally activated mechanism during the stress relaxation. The fast event has higher activation energy but is significantly biased by the work done by stress, while the slow event is less collective with a smaller activation volume, which is mainly activated by thermal energy.

\section{DISCUSSION}

\section{A. Discrete analysis of stress relaxation}

Though widely used, the stretched exponential can only describe a unimodal distribution of relaxation times with a main (or average) relaxation time. However, there is experimental evidence that several relaxation times may exist in metallic glasses [47]. The concurrent relaxation events give rise to a multimodal distribution, with well-defined relaxation times (See Fig. S6 for the generalized Maxwell model and foundation of KWW equation). These relaxation times may be different by orders of magnitude, and in this case the fit with a single stretched exponential fitting describes uniquely the dominant relaxation event.

Fig. 3 shows the results of the fit of a multimodal distribution to the relaxation data, shown in Fig. 1(a). Two well-defined relaxation times are determined below $475 \mathrm{~K}$, and three relaxation times are obtained above that temperature. In order to show the intensity at each relaxation time $\tau_{i}$, the radius of the symbol is proportional to the corresponding intensity coefficient $a_{i}$. The faster relaxation time is always lower than the threshold between the two relaxation events determined above. The characteristic time of this fast relaxation event is $\sim 10 \mathrm{~s}$, and shows a very weak temperature dependence, confirming it to be an almost instantaneous, stressdriven process. More details of discrete analysis are provided in Figs. S6 and S7 [41].

The slow relaxation times, larger than the threshold time, show a markedly different behavior above and below $670 \mathrm{~K}$. Above $670 \mathrm{~K}$, close to the glass transition temperature, both relaxation times show almost the same temperature depen- 


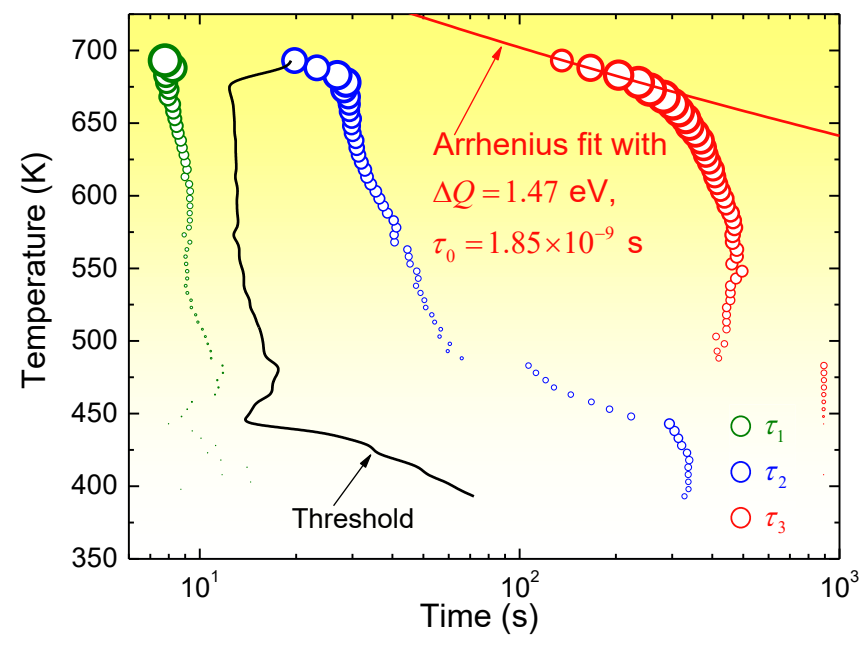

FIG. 3. (Color online) The threshold time and relaxation times $\tau_{i}$ fitted with Eq. (1). The size of the symbol is proportional to the corresponding intensity $a_{i}$.

dence, which is fitted to an Arrhenius dependence with an activation energy of $1.47 \mathrm{eV}$. It was unexpected to find two discrete relaxation times well below the glass transition. To check the validity of this fit, the "apparent" relaxation time $\tau_{\text {app }}$ was computed (Table S1 [41]). It can be seen that the "apparent" relaxation time falls in the scale of the glass transition time window. This result may not be specific to this particular metallic glass, and more experimental work is needed to check whether it appears in other, metallic or non-metallic, glasses.

Below $670 \mathrm{~K}$ both relaxation times show much weaker temperature dependence. A detailed analysis shows that this behavior is due to the length of the experimental data series. The stress relaxation data was obtained for $600 \mathrm{~s}$, and it can be seen that both relaxation times remain restricted to the experimental window. Consequently, it is deduced that the multimodal fit gives only adequate results while the relaxation times fall within the experimental window. This fact is also responsible for the vanishing of the largest relaxation time below 475 $\mathrm{K}$; below that temperature only an average relaxation time is obtained. However, a noticeable change in the slope of this average relaxation time can be seen at $450 \mathrm{~K}$, which correlates to the critical temperature of the KWW relaxation time.

\section{B. Atomistic understanding of stress relaxation}

In order to understand the microscopic mechanisms and their transition during stress relaxation, we carried out molecular dynamics (MD) simulations. The results are summarized in Fig. 4 and Fig. 5. The MD stress relaxation curves are displayed in Fig. 4(a). KWW fits are performed on those curves and the parameters are shown in Fig. 4(b). We find $\beta_{\mathrm{KWw}}$ fluctuates around 0.4 , reflecting a considerable distribution of local activation barriers during stress relaxation and resembling quantitative agreement with experiments. It is also interesting to note a variation of $\tau_{\mathrm{c}}$ with temperature similar to that found in experiment.

Note that the timescale of MD is orders of magnitude shorter than that of experiment. It could be argued that the relaxation is too fast to be representative of the macroscopic measurement; but the application of stress is also several orders of magnitude higher than in the experiment, and thus the relaxation response of the simulation is accordingly fast. Thus, the qualitative behavior or the simulations is close to that of the stress relaxation, e.g., a reasonable $\beta$ value indicating the discrete distribution of relaxation times, and the variation in $\tau_{\mathrm{c}}$ which also resembles the experimental data.

For a deeper understanding of the thermodynamic status during stress relaxation, we plot the variation of potential energy during tension and stress relaxation in Fig. 4(c). The reference potential energy is taken before loading. Tension introduces extra potential energy to the samples. But the subsequent stress relaxation process would definitely relax the glass structures energetically, and the glass continues to be aged. In particular, at higher temperature and a long enough time, the sample after stress relaxation can reach an even more relaxed state than that of the as-quenched sample. The change in the energetic state would in turn affect the stress relaxation behavior as characterized by the phenomenological KWW parameters in Fig. 1(b) and Fig. 4(b). Note that the aging effect is not quite pronounced any more at $800 \mathrm{~K}$, which is above the glass transition temperature $\left(T_{\mathrm{g}}=765 \mathrm{~K}\right.$ estimated from the present EAM potential). This is because long-range diffusion happens during stress relaxation which may interfere the energetic relaxation process.

Fig. 4(d) shows the variation of mean-squared displacement (MSD) during stress relaxation, which helps to distinguish the atomic-scale event. It has been shown that the effective jump distance of a long-range diffusion is of the order of $2 \AA$ [48], which corresponds to the MSD of $4 \AA^{2}$, as the boundary shown by a dashed line in Fig. 4(d). Fig. 4(d) indicates that most of the atoms experience a short-range displacement at lower temperature and shorter time, while long-range diffusion will occur if the time is long enough or the temperature is high enough. This atomic information indicates that the fast event is likely to be a local atomic rearrangement accommodated by hopping of energetic states between local basins, which did not contribute significantly to MSD; while the slow event has been verified by the MSD to be a longrange atomic diffusion assisted mechanism. The scenario of transition is consistent with the mode-coupling theory interpretation of diffusion as "rattling in the cage" to "cage breaking" [49-52].

\section{Competition between fast and slow event}

Fig. 5 provides the atomic-scale information about the competition between the fast and slow events, and provides a clue to recognize the distinct character of fast and slow events. Fig. 5(a) displays the distribution of the magnitude of displacements after stress relaxation for $50 \mathrm{~ns}$. The peak position $\left(d_{\text {peak }}\right)$ accounts for the most probable thermal fluctuation of all the atoms at a specific temperature. In a first approxima- 

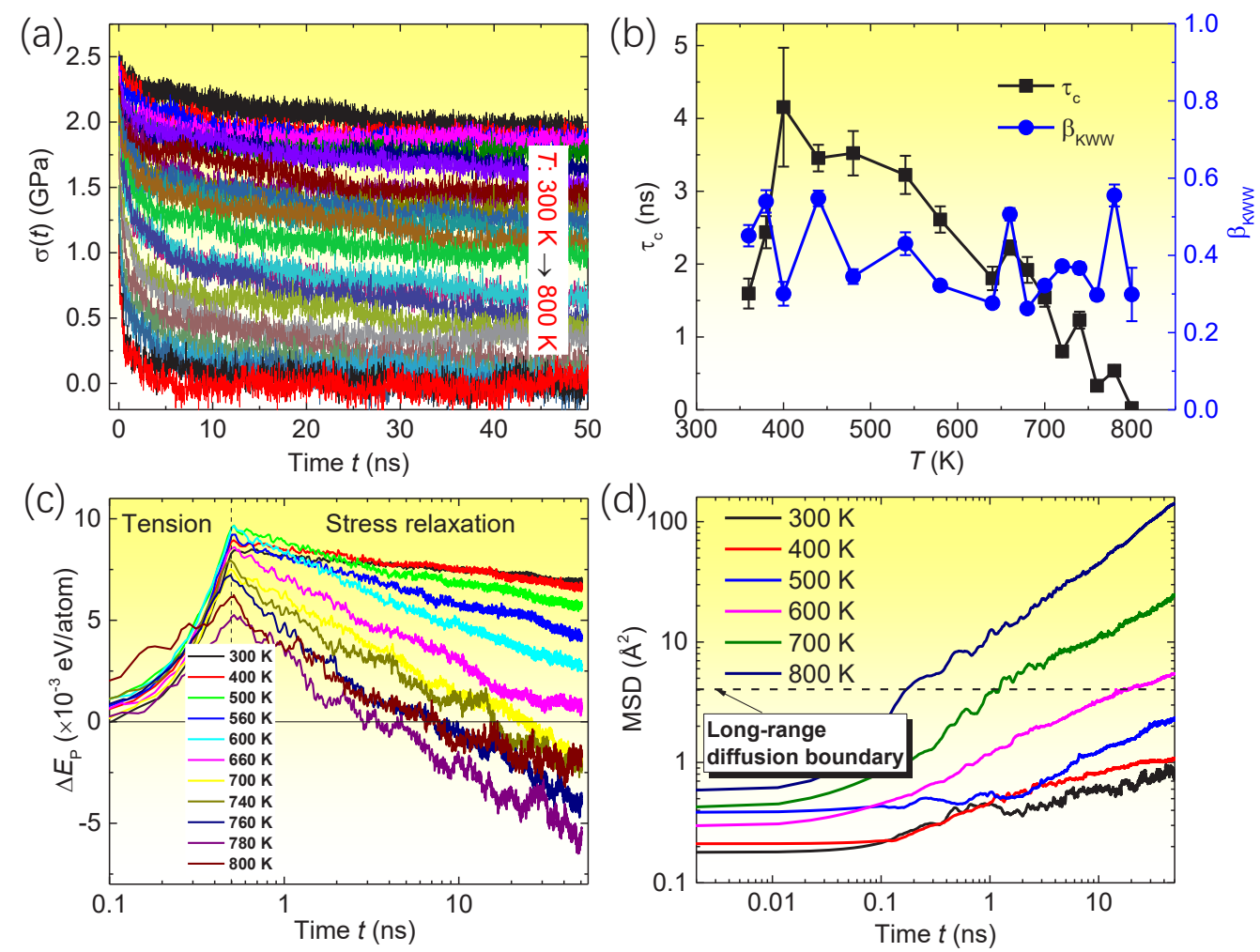

FIG. 4. (Color online) Atomistic simulations of stress relaxation in $\mathrm{Cu}_{46} \mathrm{Zr}_{46} \mathrm{Al}_{8}$. (a) The $\mathrm{MD}$ stress relaxation curves at various temperatures. (b) The KWW parameters as a function of temperature based MD. (c) Variation in potential energies accompanying the stress relaxation processes. The reference potential energies are those of the samples before loading. (d) MSD as a function of time during stress-relaxation.

tion, we categorize the atoms with displacement smaller than $2 d_{\text {peak }}$ as thermal fluctuation. Atoms exhibiting displacements between $2 d_{\text {peak }}$ and $2 \AA$ are responsible for local rearrangement and contribute to the fast event. The remaining atoms with displacement over $2 \AA$ are those that have experienced long-range diffusion. Except for the thermal fluctuation, we increasingly notice that the atoms experience long-range diffusion as denoted by the higher intensity of the curves after $2 \AA$. Particularly above $500 \mathrm{~K}$, one notices a peak for the long-range diffusive atoms. The thermal fluctuation peak vanishes at $800 \mathrm{~K}$, as expected above the glass transition where the glass transforms into an undercooled liquid.

To quantitatively rationalize the interplay between the fast and slow events, we provide the fraction of atoms participating in the local (fast) and long-range (slow) movement as a function of temperature in Fig. 5(b). The solid lines with symbols represent MD simulations. At low temperature or short time scales, the slow event dominates the stress relaxation. The fraction of the fast event shows minor temperature dependence since it is a stress-driven process, which happens in a very short time scale, while the slow event is a thermally activated event and is very sensitive to temperature. With increasing temperature, the fraction of atoms participating in the slow event increases substantially. Note that the height of the first peak in Fig. 5(a) is yet higher than that of the slow event, while the former is only the fraction of thermal fluctuation, the atoms with displacement between $2 d_{\text {peak }}$ and $2 \AA$ are the atoms participating in slow event. Up to a critical temperature of about $580 \mathrm{~K}$ in MD, the fast event dominates the stress relaxation. Note that there are several orders of magnitude between the experimental and MD time-scales; and we anticipate that the longer time scale will boost the diffusive event as the red dashed line shown in Fig. 5(b). It is reasonable to assume that the critical temperature between the fast and slow events would be much earlier in the experimental scale than in the MD simulation, as we schematically illustrate by the dashed lines in Fig. 5(b). The critical temperature of the dominating mechanism transition could happen at around 450 $\mathrm{K}$ and could rationalize the transition of $\tau_{\mathrm{c}}$ as shown in Fig. 1(b).

Last but not least, there seems to be a puzzle that why $\tau_{c}$ first increases, and then decreases, while both timescales of fast and slow events decrease monotonically with temperature. The inset of Fig. 5(b) rationalizes the transition. The effective relaxation time $\tau_{\text {eff }}$ is closely related to the participation fraction of both fast and slow events. In a first-order approximation, the effectively observable frequency of two events is expressed as, $\Gamma_{\text {eff }}(T)=\phi(T) \Gamma_{\text {fast }}(T)+[1-\phi(T)] \Gamma_{\text {slow }}(T)$, where $\phi(T)$ is the participation faction of the fast event, and $[1-\phi(T)]$ is therefore that of the slow event. The frequency is related to the relaxation time as $\Gamma=1 / \tau$, consequently, the effective relaxation time writes $\tau_{\text {eff }}=\frac{1}{\Gamma_{\text {eff }}}=\frac{\tau_{\text {fast }}(T) \tau_{\text {slow }}(T)}{\phi(T) \tau_{\text {slow }}(T)+[1-\phi(T)] \tau_{\text {fast }}(T)}$. Al- 


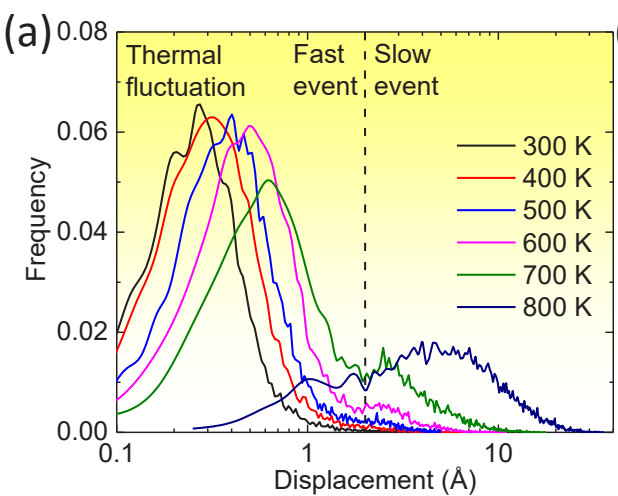

(c)

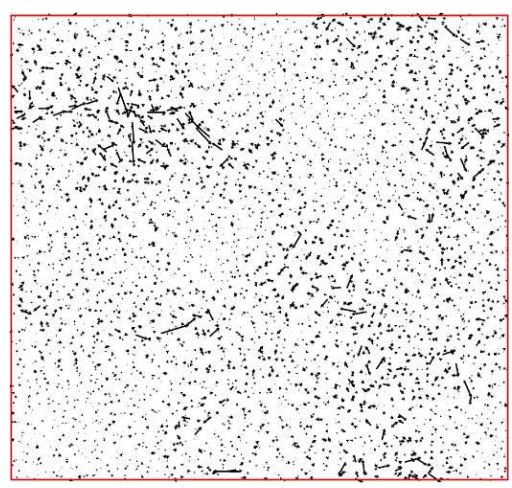

(e)

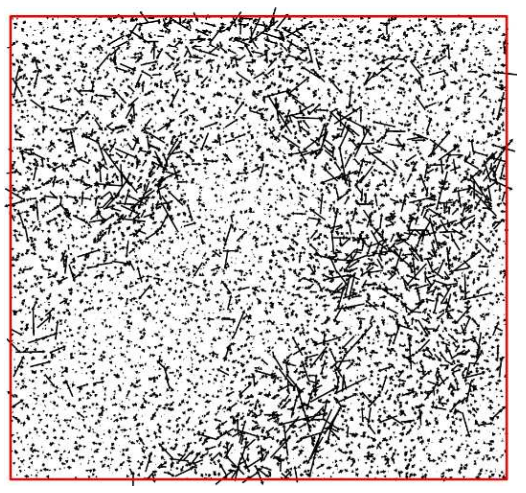

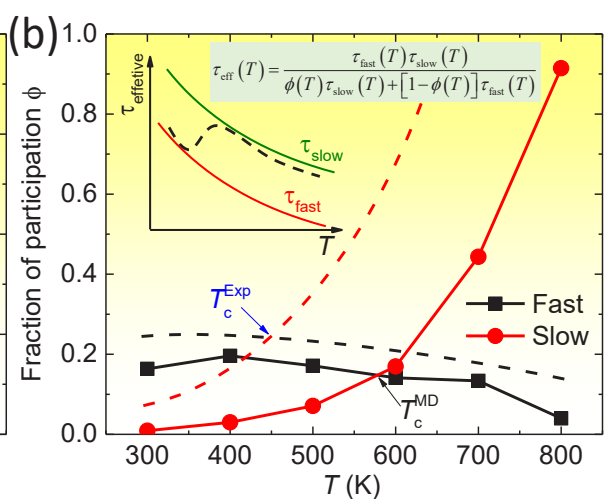

(d)

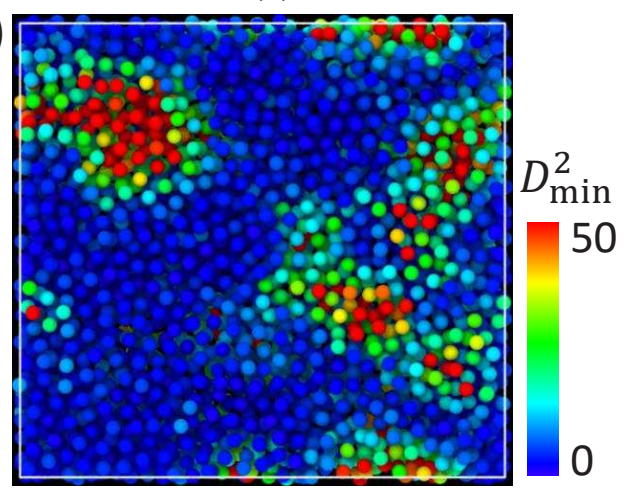

(f)

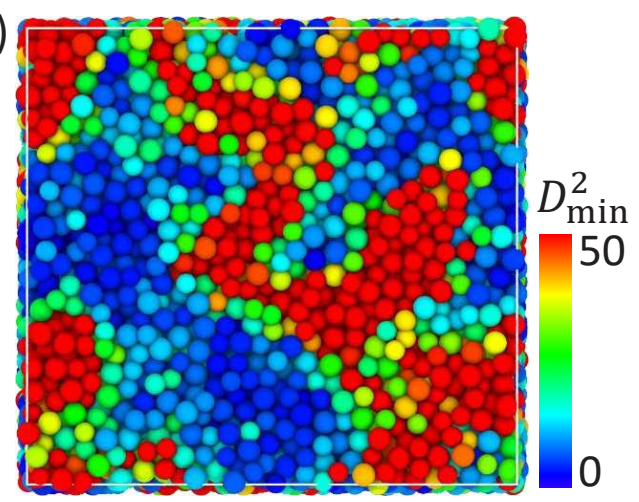

FIG. 5. (Color online) Competition between the local atomic rearrangement and the long-ranged diffusive mechanism during stress relaxation. (a) Distribution of displacement at different temperatures after stress-relaxation for $50 \mathrm{~ns}$. The fast and slow event are defined by this plot. (b) Faction of the atoms participating in fast and slow event as a function of temperature. Solid lines with symbols are the MD data, while the dashed lines are the schematic speculations at the experimental time-scale. The inset is a sketch illustrating the variation of effective characteristic time with temperature. (c) and (e) Displacement vector, (d) and (f) non-affine squared displacement of the stress-relaxed samples after $50 \mathrm{~ns}$ at $300 \mathrm{~K}$, and $500 \mathrm{~K}$, respectively. Only the atoms in a slice of $3 \mathrm{~nm}$ are shown for clarity. Fast event involves local collective atomic rearrangement, while the slow event is the percolation of the former accommodated by the long-range atomic diffusion.

though both $\tau_{\text {fast }}(T)$ and $\tau_{\text {slow }}(T)$ decrease with increasing temperature, $\phi(T)$ decreases simultaneously [as shown in Fig. 5(b)], which means that the effective relaxation time $\tau_{\text {eff }}(T)$ transitions from $\tau_{\text {fast }}(T)$ to $\tau_{\text {slow }}(T)$ in the two limits of $\phi(T)=1$, or $\phi(T)=0$. Therefore, the decrease of $\phi(T)$ with $T$ induces the increase of $\tau_{\text {eff }}$ at intermediate temperatures as the slow events eventually dominate the relaxation dynamics at high temperature, as schematically illustrated in the inset of Fig. 5(b).

Finally, the atomistic simulations provide scenarios of both the fast and slow events, as demonstrated in Fig. 5(c-f).
Fig. 5(c-d) display the displacement vector and the non-affine squared displacement $D_{\min }^{2}(42)$ of the snapshot after stress relaxation for $50 \mathrm{~ns}$ at $300 \mathrm{~K}$. They correspond to the fast event. The displacement vector indicates local motion of atoms in a confined volume of the order of nanometers and involves local rearrangement of a large number of atoms as indicated by $D_{\min }^{2}$ and the large activation volume shown in Fig. 2(b). On the other hand, Fig. 5(e-f) show the counterparts for the slow event. In contrast to the fast event, the slow event requires a long-range diffusion. It is representative of the behavior of very few atoms characterized by the smaller activation vol- 
ume. The atomic scenario of the slow event is actually the percolation of the local atomic rearrangement with a critical role of atomic diffusion [35, 53, 54], which is a thermally activated process at a low stress level that needs a longer time to be excited. The above scenario of the local atomic rearrangement and the long-range diffusion in stress relaxation is in accordance with the hypothesized atomic pictures of a recently discovered unusual ultrafast and usual fast $\beta$ relaxation in MGs [55]. Here we further provide an atomic-scale evidence for the fast stress-driven, and slow thermally activated processes during stress relaxation of MGs.

\section{CONCLUDING REMARKS}

To summarize, a mechanism transition from stress-driven, to thermally activated stress relaxation in metallic glass is unveiled by a combined study of experiment, theory, and computer simulations. The stress-driven event is fast and characteristic of large activation volume which dominates the early stage of stress relaxation. The relaxation mechanism gradually transitions to slow mechanism which is governed by the thermally-activated mechanism that is typical of small activation volume. Discrete analysis distinguishes the heterogenous dynamics and rationalizes the transition of stress relaxation mechanisms. Atomistic simulations demonstrate the interplay between fast and slow event and display similar results with experimental observations. Simulations reveal the atomic-scale mechanism of fast event as the local atomic rearrangement, while the slow event is the percolation of the former involving a significant contribution from the atomic diffusion, which needs longer timescale to occur. The combined insights provide comprehensive physical insights into the underlying mechanisms of the phenomenological KWW equation and may shed light on elucidating the mechanism of the percolation of the secondary $\beta$ relaxation to primary $\alpha$ relaxation in glassy materials.

\section{ACKNOWLEDGMENTS}

This work is partially supported by the NSFC (Grant Nos. 11132011, 11402269, 11472287, 51401192, 11572249, 51611130120), the National Key Basic Research Program of China (Grant No. 2012CB937500), the Natural Science Foundation of Shaanxi Province (No. 2016JM5009), the Fundamental Research Funds for the Central Universities (No. 3102015ZY027, 3102015BJ(II)JGZ019), the Aeronautical Science Foundation of China (2015ZF53072), Opening fund of State Key Laboratory of Nonlinear Mechanics (LNM201511), and the CAS/SAFEA International Partnership Program for Creative Research Teams. D.C. acknowledges the support from Spanish MINECO (Grant FIS2014-54734-P) and Generalitat de Catalunya (Grant 2014SGR00581).
[1] P. G. Debenedetti and F. H. Stillinger, "Supercooled liquids and the glass transition," Nature 410, 259-267 (2001).

[2] W. L. Johnson, M. D. Demetriou, J. S. Harmon, M. L. Lind, and K. Samwer, "Rheology and Properties of Metallic GlassForming Liquids : A Potential Energy Landscape Perspective," MRS Bull. 32, 644-650 (2007).

[3] W. H. Wang, "The elastic properties, elastic models and elastic perspectives of metallic glasses," Prog. Mater. Sci. 57, 487-656 (2012).

[4] C. A. Angell, "Formation of glasses from liquids and biopolymers." Science 267, 1924-1935 (1995).

[5] H. W. Sheng, W. K. Luo, F. M. Alamgir, J. M. Bai, and E. Ma, "Atomic packing and short-to-medium-range order in metallic glasses." Nature 439, 419-425 (2006).

[6] J. Ding, S. Patinet, M. L. Falk, Y. Cheng, and E. Ma, "Soft spots and their structural signature in a metallic glass," Proc. Natl. Acad. Sci. U. S. A. 111, 14052-14056 (2014).

[7] D. Pan, A. Inoue, T. Sakurai, and M. W. Chen, "Experimental characterization of shear transformation zones for plastic flow of bulk metallic glasses." Proc. Natl. Acad. Sci. U. S. A. 105, 14769-14772 (2008).

[8] D. Z. Chen, C. Y. Shi, Q. An, Q. S. Zeng, W. L. Mao, Goddard III W. A., and J. R. Greer, "Fractal atomic-level percolation in metallic glasses," Science 349, 1306-1310 (2015).

[9] W. Dmowski, T. Iwashita, C. P. Chuang, J. Almer, and T. Egami, "Elastic heterogeneity in metallic glasses," Phys. Rev. Lett. 105, 205502 (2010).

[10] J. C. Ye, J. Lu, C. T. Liu, Q. Wang, and Y. Yang, "Atomistic free-volume zones and inelastic deformation of metallic glasses." Nat. Mater. 9, 619-623 (2010).

[11] T. Kawasaki, T. Araki, and H. Tanaka, "Correlation between Dynamic Heterogeneity and Medium-Range Order in Two-Dimensional Glass-Forming Liquids," Phys. Rev. Lett. 99, 215701 (2007).

[12] Y. H. Liu, D. Wang, K. Nakajima, W. Zhang, A. Hirata, T. Nishi, A. Inoue, and M. W. Chen, "Characterization of nanoscale mechanical heterogeneity in a metallic glass by dynamic force microscopy," Phys. Rev. Lett. 106, 125504 (2011).

[13] J. H. Perepezko, S. D. Imhoff, M. W. Chen, J. Q. Wang, and S. Gonzalez, "Nucleation of shear bands in amorphous alloys." Proc. Natl. Acad. Sci. U. S. A. 111, 3938-3942 (2014).

[14] C. Bennemann, C. Donati, J. Baschnagel, and S. C. Glotzer, "Growing range of correlated motion in a polymer melt on cooling towards the glass transition," Nature 399, 246-249 (1999).

[15] A. J. Liu and S. R. Nagel, "Nonlinear dynamics: Jamming is not just cool any more," Nature 396, 21-22 (1998).

[16] J. Krausser, K. H. Samwer, and A. Zaccone, "Interatomic repulsion softness directly controls the fragility of supercooled metallic melts," Proc. Natl. Acad. Sci. U. S. A. 112, 13762 13767 (2015).

[17] S. S. Schoenholz, A. J. Liu, R. A. Riggleman, and J. Rottler, "Understanding Plastic Deformation in Thermal Glasses from Single-Soft-Spot Dynamics," Phys. Rev. X 4, 031014 (2014).

[18] S. S. Schoenholz, E. D. Cubuk, D. M. Sussman, E. Kaxiras, and A. J. Liu, "A structural approach to relaxation in glassy liquids," Nat. Phys. 12, 469-471 (2016).

[19] B. Zhang and X. Cheng, "Structures and dynamics of glassforming colloidal liquids under spherical confinement," Phys. 
Rev. Lett. 116, 098302 (2016).

[20] C. P. Royall, S. R. Williams, T. Ohtsuka, and H. Tanaka, "Direct observation of a local structural mechanism for dynamic arrest." Nat. Mater. 7, 556-561 (2008).

[21] R. L. Jack, A. J. Dunleavy, and C. P. Royall, "Informationtheoretic measurements of coupling between structure and dynamics in glass formers," Phys. Rev. Lett. 113, 095703 (2014).

[22] F. Zhu, H. K. Nguyen, S. X. Song, D. P. B. Aji, A. Hirata, H. Wang, K. Nakajima, and M. W. Chen, "Intrinsic correlation between $\beta$-relaxation and spatial heterogeneity in a metallic glass," Nat. Commun. 7, 11516 (2016).

[23] Y. Q. Cheng and E. Ma, "Atomic-level structure and structureproperty relationship in metallic glasses," Prog. Mater. Sci. 56, 379-473 (2010).

[24] S. V. Ketov, Y. H. Sun, S. Nachum, Z. Lu, A. Checchi, A. R. Beraldin, H. Y. Bai, W. H. Wang, D. V. Louzguine-Luzgin, M. A. Carpenter, and A. L. Greer, "Rejuvenation of metallic glasses by non-affine thermal strain," Nature 524, 200-203 (2015).

[25] M. Wakeda, J. Saida, J. Li, and S. Ogata, "Controlled Rejuvenation of Amorphous Metals with Thermal Processing," Sci. Rep. 5, 10545 (2015).

[26] C. Schuh, T. Hufnagel, and U. Ramamurty, "Mechanical behavior of amorphous alloys," Acta Mater. 55, 4067-4109 (2007).

[27] A. L. Greer, Y. Q. Cheng, and E. Ma, "Shear bands in metallic glasses," Mater. Sci. Eng. R Reports 74, 71-132 (2013).

[28] C. C. Wang, Y. W. Mao, Z. W. Shan, M. Dao, J. Li, J. Sun, E. Ma, and S. Suresh, "Real-time, high-resolution study of nanocrystallization and fatigue cracking in a cyclically strained metallic glass.” Proc. Natl. Acad. Sci. U. S. A. 110, 19725-30 (2013).

[29] T. C. Hufnagel, C. A. Schuh, and M. L. Falk, "Deformation of metallic glasses: Recent developments in theory, simulations, and experiments," Acta Mater. 109, 375-393 (2016).

[30] Z. Wang, B. A. Sun, H. Y. Bai, and W. H. Wang, "Evolution of hidden localized flow during glass-to-liquid transition in metallic glass," Nat. Commun. 5, 5823 (2014).

[31] Z. Lu, W. Jiao, W. H. Wang, and H. Y. Bai, "Flow Unit Perspective on Room Temperature Homogeneous Plastic Deformation in Metallic Glasses," Phys. Rev. Lett. 113, 045501 (2014).

[32] Y. Z. Li, L. Z. Zhao, C. Wang, Z. Lu, H. Y. Bai, and W. H. Wang, "Communication: Non-monotonic evolution of dynamical heterogeneity in unfreezing process of metallic glasses," J. Chem. Phys. 143, 041104 (2015).

[33] J. C. Qiao, Y. J. Wang, J. M. Pelletier, L. M. Keer, M. E. Fine, and Y. Yao, "Characteristics of stress relaxation kinetics of $\mathrm{La}_{60} \mathrm{Ni}_{15} \mathrm{Al}_{25}$ bulk metallic glass," Acta Mater. 98, 43-50 (2015).

[34] Z. Lu, W. H. Wang, and H. Y. Bai, "Classification of metallic glasses based on structural and dynamical heterogeneities by stress relaxation," Sci. China Mater. 58, 98-105 (2015).

[35] H. B. Yu, K. Samwer, Y. Wu, and W. H. Wang, "Correlation between $\beta$ Relaxation and Self-Diffusion of the Smallest Constituting Atoms in Metallic Glasses," Phys. Rev. Lett. 109, 095508 (2012).
[36] Y. H. Liu, T. Fujita, D. P. B. Aji, M. Matsuura, and M. W. Chen, "Structural origins of Johari-Goldstein relaxation in a metallic glass." Nat. Commun. 5, 3238 (2014).

[37] Maple is a trademark of Waterloo Maple Inc.

[38] S. Plimpton, "Fast Parallel Algorithms for Short-Range Molecular Dynamics," J. Comput. Phys. 117, 1-19 (1995).

[39] Y. Q. Cheng, E. Ma, and H. W. Sheng, "Atomic Level Structure in Multicomponent Bulk Metallic Glass,” Phys. Rev. Lett. 102, 245501 (2009).

[40] A. Stukowski, "Visualization and analysis of atomistic simulation data with OVITO-the Open Visualization Tool," Model. Simul. Mater. Sci. Eng. 18, 015012 (2010).

[41] See Supplemental Materials for extra experimental data, and details of theories.

[42] Y. Fan, T. Iwashita, and T. Egami, "How thermally activated deformation starts in metallic glass," Nat. Commun. 5, 5083 (2014).

[43] Y. Fan, T. Iwashita, and T. Egami, "Crossover from Localized to Cascade Relaxations in Metallic Glasses," Phys. Rev. Lett. 115, 045501 (2015).

[44] T. Zhu, J. Li, A. Samanta, A. Leach, and K. Gall, "Temperature and Strain-Rate Dependence of Surface Dislocation Nucleation," Phys. Rev. Lett. 100, 025502 (2008).

[45] Y. Fan, Y. Osetsky, S. Yip, and B. Yildiz, "Onset Mechanism of Strain-Rate-Induced Flow Stress Upturn,” Phys. Rev. Lett. 109, 135503 (2012).

[46] H. B. Yu, W. H. Wang, H. Y. Bai, Y. Wu, and M. W. Chen, "Relating activation of shear transformation zones to $\beta$ relaxations in metallic glasses," Phys. Rev. B(R) 81, 220201 (2010).

[47] J. D. Ju, D. Jang, A. Nwankpa, and M. Atzmon, “An atomically quantized hierarchy of shear transformation zones in a metallic glass," J. Appl. Phys. 109, 053522 (2011).

[48] X. P. Tang, U. Geyer, R. Busch, W. L. Johnson, and Y. Wu, "Diffusion mechanisms in metallic supercooled liquids and glasses," Nature 402, 160-162 (1999).

[49] H. Teichler, "Heterogeneous dynamics on the microsecond scale in simulated $\mathrm{Ni}_{0.5} \mathrm{Zr}_{0.5}$ metallic melts far below the glass temperature,” Phys. Rev. E 71, 031505 (2005).

[50] P. I. Hurtado, L. Berthier, and W. Kob, "Heterogeneous diffusion in a reversible gel," Phys. Rev. Lett. 98, 135503 (2007).

[51] K. Vollmayr-Lee, T. Aspelmeier, and A. Zippelius, "Hydrodynamic correlation functions of a driven granular fluid in steady state," Phys. Rev. E 83, 011301 (2011).

[52] D. Winter, J. Horbach, P. Virnau, and K. Binder, "Active nonlinear microrheology in a glass-forming Yukawa fluid," Phys. Rev. Lett. 108, 028303 (2012).

[53] Y. F. Shi and M. L. Falk, "Strain localization and percolation of stable structure in amorphous solids," Phys. Rev. Lett. 95, 095502 (2005).

[54] P. Luo, Z. Lu, Y. Z. Li, H. Y. Bai, P. Wen, and W. H. Wang, "Probing the evolution of slow flow dynamics in metallic glasses," Phys. Rev. B 93, 104204 (2016).

[55] Q. Wang, S. T. Zhang, Y. Yang, Y. D. Dong, C. T. Liu, and J. Lu, "Unusual fast secondary relaxation in metallic glass," Nat. Commun. 6, 7876 (2015). 